\title{
Improved Reception of In-Body Signals by Means of a Wearable Multi-Antenna System
}

\author{
Thijs Castel, ${ }^{1}$ Patrick Van Torre, ${ }^{1,2}$ Emmeric Tanghe, ${ }^{1}$ Sam Agneessens, ${ }^{1}$ \\ Günter Vermeeren, ${ }^{1}$ Wout Joseph, ${ }^{1}$ and Hendrik Rogier ${ }^{1}$ \\ ${ }^{1}$ Department of Information Technology, INTEC-IMEC, Ghent University, Sint-Pietersnieuwstraat 41, 9000 Ghent, Belgium \\ ${ }^{2}$ Department IT\&C, GEN Group, Ghent University, Valentin Vaerwyckweg 1, 9000 Ghent, Belgium
}

Correspondence should be addressed to Thijs Castel; thijs.castel@ugent.be

Received 5 April 2013; Revised 16 July 2013; Accepted 17 July 2013

Academic Editor: Lorenzo Luini

Copyright (C) 2013 Thijs Castel et al. This is an open access article distributed under the Creative Commons Attribution License, which permits unrestricted use, distribution, and reproduction in any medium, provided the original work is properly cited.

High data-rate wireless communication for in-body human implants is mainly performed in the $402-405 \mathrm{MHz}$ Medical Implant Communication System band and the $2.45 \mathrm{GHz}$ Industrial, Scientific and Medical band. The latter band offers larger bandwidth, enabling high-resolution live video transmission. Although in-body signal attenuation is larger, at least $29 \mathrm{~dB}$ more power may be transmitted in this band and the antenna efficiency for compact antennas at $2.45 \mathrm{GHz}$ is also up to 10 times higher. Moreover, at the receive side, one can exploit the large surface provided by a garment by deploying multiple compact highly efficient wearable antennas, capturing the signals transmitted by the implant directly at the body surface, yielding stronger signals and reducing interference. In this paper, we implement a reliable $3.5 \mathrm{Mbps}$ wearable textile multi-antenna system suitable for integration into a jacket worn by a patient, and evaluate its potential to improve the In-to-Out Body wireless link reliability by means of spatial receive diversity in a standardized measurement setup. We derive the optimal distribution and the minimum number of on-body antennas required to ensure signal levels that are large enough for real-time wireless endoscopy-capsule applications, at varying positions and orientations of the implant in the human body.

\section{Introduction}

Many studies about global aging show an increasing life expectancy in about every continent $[1,2]$. This trend is expected to continue in the near future, necessitating a renewed vision on medical healthcare. By enabling wireless communication from inside the body towards the outside world (in-to-out body communication), a whole range of new possibilities arise. In particular, wireless capsule endoscopy (WCE) [3] is an important but technically demanding application. Current commercial WCE systems (Given Imaging, Olympus EndoCapsule) propagate passively through the intestine and operate at low frame rate $[4,5]$. Uncontrolled orientation and movement as well as low resolution are considered a major drawback of passive systems [6-8]. This resulted in research towards actively controlled capsules, requiring higher frame rates and resolution to provide realtime video feedback to the physician steering the capsule movement to interactively focus on diagnostically important features $[9,10]$.

Frequency bands commonly used for wireless implant links are the 402-405 medical implant communication system (MICS) band (or the 401-406 MedRadio band [11]) as well as the $2.45 \mathrm{GHz}$ industrial, scientific, and medical (ISM) band. The maximum available bandwidth per channel in the MICS band is $300 \mathrm{kHz}$ [12], in contrast to the much larger channel bandwidth of $20 \mathrm{MHz}$ in the ISM band [13]. Therefore, the $2.45 \mathrm{GHz}$ band is more suitable for WCE with high-resolution live video transmission $[14,15]$. The use of ultrawide band (UWB) technology is proposed for an in-to-out body link suggesting high data rates, low power consumption, and simple electronics [16-20]. However, only channel modeling and characterization are described. To our knowledge, no measurements were performed. UWB with diversity is proposed in $[21,22]$ documenting a theoretical 
approach based on simulations. Practically, UWB applications are mostly limited to implants requiring low signal penetration depth, such as cortical implants [23] due to the very high signal attenuation and the limited available transmit power [24]. As the in-body attenuation at $2.45 \mathrm{GHz}$ is smaller than in the $3.4-4.8 \mathrm{GHz}$ low-UWB band [25], the ISM band combines sufficient bandwidth with acceptable attenuation.

We propose a novel reliable high date-rate wearable higher-order diversity in-to-out body communication system to fully compensate for the higher in-body attenuation at $2.45 \mathrm{GHz}$ by mitigating this attenuation using multiple wearable antennas around the body. Diversity systems are realistic in the ISM band, thanks to the much shorter wavelength and smaller dimensions of electrically full-size antennas, compared to the MICS band. Hence, there is enough space for a wearable multiantenna system on the human body or in a garment. The proposed diversity system enables the use of reliable wide-bandwidth/high-data rate wireless implant links in the $2.45 \mathrm{GHz}$ ISM band. The effect of $4 \mathrm{th}$, 6th, and 8th order spatial diversity is examined for an $x-, y$ - and $z$ oriented dipole and for different positions of the wearable antennas capturing the signals transmitted by the implant at the surface of a human body phantom.

In addition to applying diversity, the larger in-body attenuation present at $2.45 \mathrm{GHz}$ is partially compensated for by a three orders of magnitude higher permitted transmit power in the ISM band. Regulatory standards limit the (in-body) radiation to $25 \mu \mathrm{W}$ (-16 dBm EIRP) in the MICS band [26]. In the $2.45 \mathrm{GHz}$ ISM band, the European Telecommunications Standards Institute (ETSI) limits transmit power to $100 \mathrm{~mW}$ (20 dBm EIRP). In addition, for the same ISM band an SAR limit of $2 \mathrm{~W} / \mathrm{kg}$ averaged over $10 \mathrm{~g}$ tissue is specified by IEC 62209 [27]. Even if all RF-energy would be absorbed by the $10 \mathrm{~g}$ tissue directly surrounding the antenna, $20 \mathrm{~mW}(13 \mathrm{dBm}$ EIRP) is allowed, resulting in at least $29 \mathrm{~dB}$ more available transmit power in the $2.45 \mathrm{GHz}$ ISM band, compared to the MICS band. Specifically for steerable endoscopy capsules, the ISM band is a good candidate thanks to the larger bandwidth for the high data rate video downlink, combined with a possible MICS band uplink for controlling the capsule without mutual interference.

Additionally, thanks to the shorter wavelength, compact yet electrically full-size in-body antennas yield up to $10 \mathrm{~dB}$ additional antenna gain for the $2.45 \mathrm{GHz}$ ISM band [28, 29]. Multiple compact on-body antennas enable receive diversity. Moreover, high-speed wireless communication standards are commonly implemented in integrated circuits for this band, resulting in readily available system components. These two advantages leverage the design of compact low-power as well as low-cost WCE devices.

In-to-out body communication is an active research topic, and important previous work was performed by many research groups. A detailed analysis of wave propagation and radiation efficiency in different human tissues (such as lungs, stomach, liver, heart, skin, and muscle), at different frequencies ( $402 \mathrm{MHz}, 868 \mathrm{MHz}$, and $2.45 \mathrm{GHz}$ ) is presented in [30-33]. A number of advantages of using the 2.45 ISM band instead of the 402-405 MICS band are described in [31]. In [34], a comparison between the body worn antenna efficiency and pattern fragmentation at $418 \mathrm{MHz}$ and $916.5 \mathrm{MHz}$ is presented. Moreover, research on an in-toout body communication link through human muscle tissue in the $2.45 \mathrm{GHz}$ ISM band is presented in $[35,36]$, where path-loss models are derived, with and without inclusion of the antenna gain, respectively. Transceiver development for medical endoscopy applications (ISM band) and implantable devices (MICS-band) is presented in $[15,37]$, respectively, focusing on low power and high-data rates.

To evaluate the performance of our system, we rely on a standardized phantom, compatible with the IEC 62209 standard within the frequency range $30 \mathrm{MHz}-6 \mathrm{GHz}$, as a means to assess system performance for a person of average size and weight. It is well known that different types of body tissue have varying conductance and permittivity. Yet, muscle tissue $\left(\sigma_{m}=1.7388 \mathrm{~S} / \mathrm{m}\right.$ [38]) causes the largest signal attenuation at $2.45 \mathrm{GHz}$. Therefore, the phantom was filled with muscle-simulating liquid to validate the channel in worst-case propagation conditions in an average body size. Recommendations of [29] were followed for human body modeling by using muscle-like dielectric properties, providing standard and easy to reproduce measurements conditions.

The paper is further organized as follows. Section 2 describes the wearable antenna system and the measurement setup. Section 3 details the results of the different experiments, examining the performance of different diversity schemes. Finally, the conclusions are presented in Section 4.

\section{Materials and Methods}

2.1. Construction of the Wearable Antenna System. A wearable multiantenna system for integration into a jacket was developed, as presented in Figure 1. It consists of a set of wearable on-body textile patch antennas, distributed such that they cover different areas of the body and oriented towards the body to capture the signals transmitted by an inbody implant.

The on-body receiving textile patch antennas are designed to be matched to and radiate into the human body, instead of radiating in free space away from the human body [39]. These antennas exhibit a stable performance for changing parameters such as different body morphologies, movement of the patient, and varying electrical parameters of different organs. The rectangular ring antenna topology with probe feed is shown in Figure 2. Its dimensions are described in the caption of this figure. The antenna substrate consists of very flexible closed-cell expanded protective foam $\left(\varepsilon_{r}=1.485, \tan \delta=0.0243\right)$, and the ground plane and the patch are fabricated using the $80 \mu \mathrm{m}$-thick e-textile Flectron (sheet resistivity $0.18 \Omega / \mathrm{sq}$ at $2.45 \mathrm{GHz}$ ). The foam spacer, used to physically separate the body from the conductive part of the antenna and ensuring $50 \Omega$ matching in all conditions, is fabricated by means of a foam layer with thickness $h_{2}=7.92 \mathrm{~mm}$.

By integrating several such on-body receive antennas at suitable locations into a jacket, we obtain a multiantenna 
TABLE 1: Human body phantom dimensions (in $\mathrm{cm}$ ).

\begin{tabular}{|c|c|c|c|c|c|c|c|}
\hline \multicolumn{6}{|c|}{ Human body phantom dimensions (cm) } & \multicolumn{2}{|c|}{ Scan points $(x, y)$} \\
\hline$W_{1}$ & 65 & $\mathrm{~A}$ & 8.5 & $\mathrm{~A}^{\prime}$ & 31 & $\mathrm{P} 1$ & $(0,0)$ \\
\hline$W_{2}$ & 59 & B & 5.2 & $\mathrm{~B}^{\prime}$ & 17.6 & $\mathrm{P} 2$ & $(385,0)$ \\
\hline$L_{1}$ & 41.5 & $\mathrm{C}$ & 5.1 & $\mathrm{C}^{\prime}$ & 14.9 & P3 & $(0,215)$ \\
\hline$L_{2}$ & 39 & $\mathrm{D}$ & 6.5 & $\mathrm{D}^{\prime}$ & 13.8 & P4 & $(385,215)$ \\
\hline$h$ & 18.7 & $\mathrm{E}$ & 9.3 & $\mathrm{E}^{\prime}$ & 31 & P5 & $(192,105)$ \\
\hline$h_{v 1}$ & 10.2 & $\mathrm{~F}$ & 7.5 & $\mathrm{~F}^{\prime}$ & 19.9 & P6 & $(385,105)$ \\
\hline$S_{w}$ & 11 & G & 9.5 & $\mathrm{G}^{\prime}$ & 17.2 & P7 & $(192,150)$ \\
\hline$S_{b 1}$ & 15 & $\mathrm{H}$ & 13.8 & $\mathrm{H}^{\prime}$ & 13.8 & P8 & $(385,150)$ \\
\hline$S_{b 2}$ & 13 & & & & & & \\
\hline
\end{tabular}

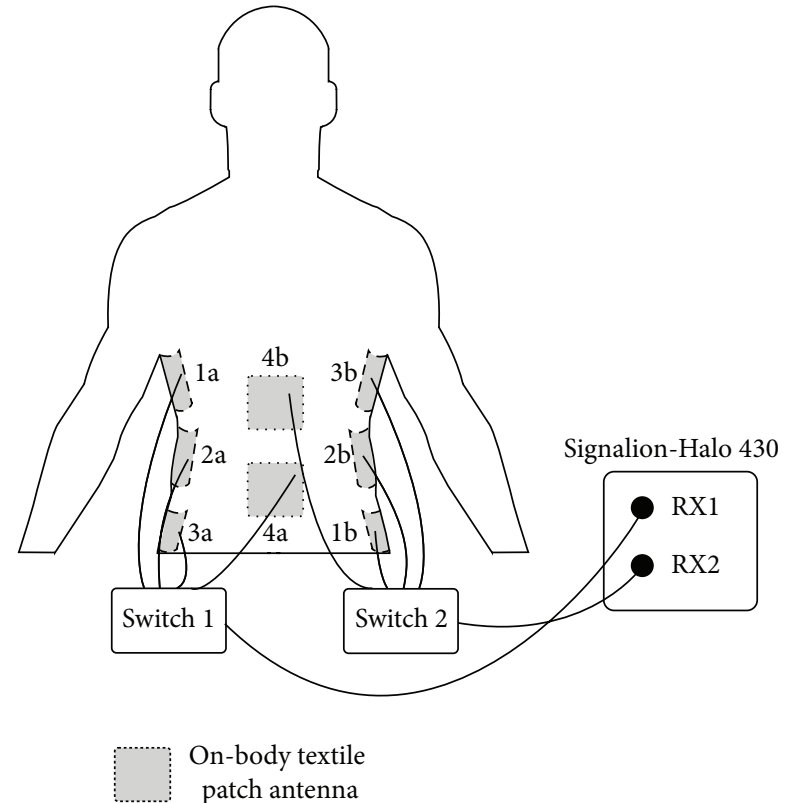

FIgURE 1: Wearable multiantenna system, with 6 side (1a, 2a, 3a, 1b, $2 \mathrm{~b}, 3 \mathrm{~b})$ and 2 back (4a, 4b) antennas, deployed on a patient and connected to the Signalion-HaLo 430 by means of two switches.

system enabling highly reliable broadband data communication with compact low-power implants. We set the critical level required for highly reliable live wireless video streaming to $10 \mathrm{~dB}$ received signal-to-noise ratio (SNR). Given the Shannon-Hartley theorem, this critical level corresponds to a minimal bitrate of $3.5 \mathrm{Mbit} / \mathrm{s}$ within a bandwidth of $1 \mathrm{MHz}$, which is sufficient for live wireless video streaming, for example, in wireless endoscopy-capsule applications [40], this without the necessity of data buffering and thereby data retransmission, still ensuring correct reception of the inbody signals for all potential positions and orientations of the implant in the human body.

2.2. Measurement Setup. Figure 3 depicts the measurement setup, and Table 1 lists the dimensions of the human body phantom (in $\mathrm{cm}$ ). The coordinates of the points $\mathrm{P} 1$ to $\mathrm{P} 8$ are indicated as $(x, y)$.

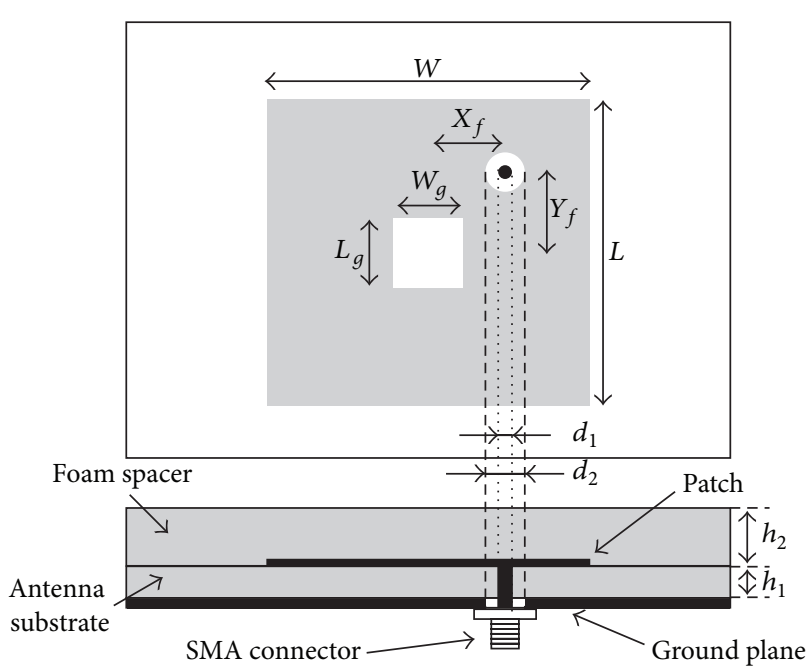

Figure 2: Patch antenna topology $(W=40.9 \mathrm{~mm}, L=48.7 \mathrm{~mm}$, $W_{g}=8.8 \mathrm{~mm}, L_{g}=13.2 \mathrm{~mm}, X_{f}=7.8 \mathrm{~mm}, Y_{f}=18.5 \mathrm{~mm}, h_{1}=$ $\left.3.94 \mathrm{~mm}, h_{2}=7.92 \mathrm{~mm}, d_{1}=1.3 \mathrm{~mm}, d_{2}=5.5 \mathrm{~mm}\right)$.

In the measurement setup, the implant is represented by an insulated half-wavelength dipole [35] resonating at $2.457 \mathrm{GHz}$ as transmit antenna. The dipole is coated by an insulation of polytetrafluorethylene $\left(\varepsilon_{\mathrm{r}}=2.07\right.$ and $\sigma=$ $0 \mathrm{~S} / \mathrm{m}$ ), as shown in Figure 4.

The human body is simulated by an oval ELI flat phantom, fabricated by Speag (Zürich, Switzerland), compatible with the IEC 62209 standard within the frequency range $30 \mathrm{MHz}-$ $6 \mathrm{GHz}$. This flat phantom is filled with (MSL2450) human muscle tissue mimicking liquid (relative permittivity $\varepsilon_{r}=$ 50.8 , conductivity $\sigma_{m}=2.01 \mathrm{~S} / \mathrm{m}$ ). Two $50 \Omega \mathrm{SP} 4 \mathrm{~T}$ pindiode switches (Mini-Circuits ZSDR-425) select the signals, received from the different patch antennas, to be forwarded to the Signalion-HaLo 430 measurement test bed, interfacing to Matlab. A loopback connection is provided to guarantee reliable timing synchronization during postprocessing. Details of the transmitted signals and postprocessing are described in [41].

Measurements were then performed for varying polarizations of the insulated dipole antenna: an $x$-oriented horizontal polarization, a $y$-oriented horizontal polarization, and a vertical polarization as also used in [39]. For the $x$ and 

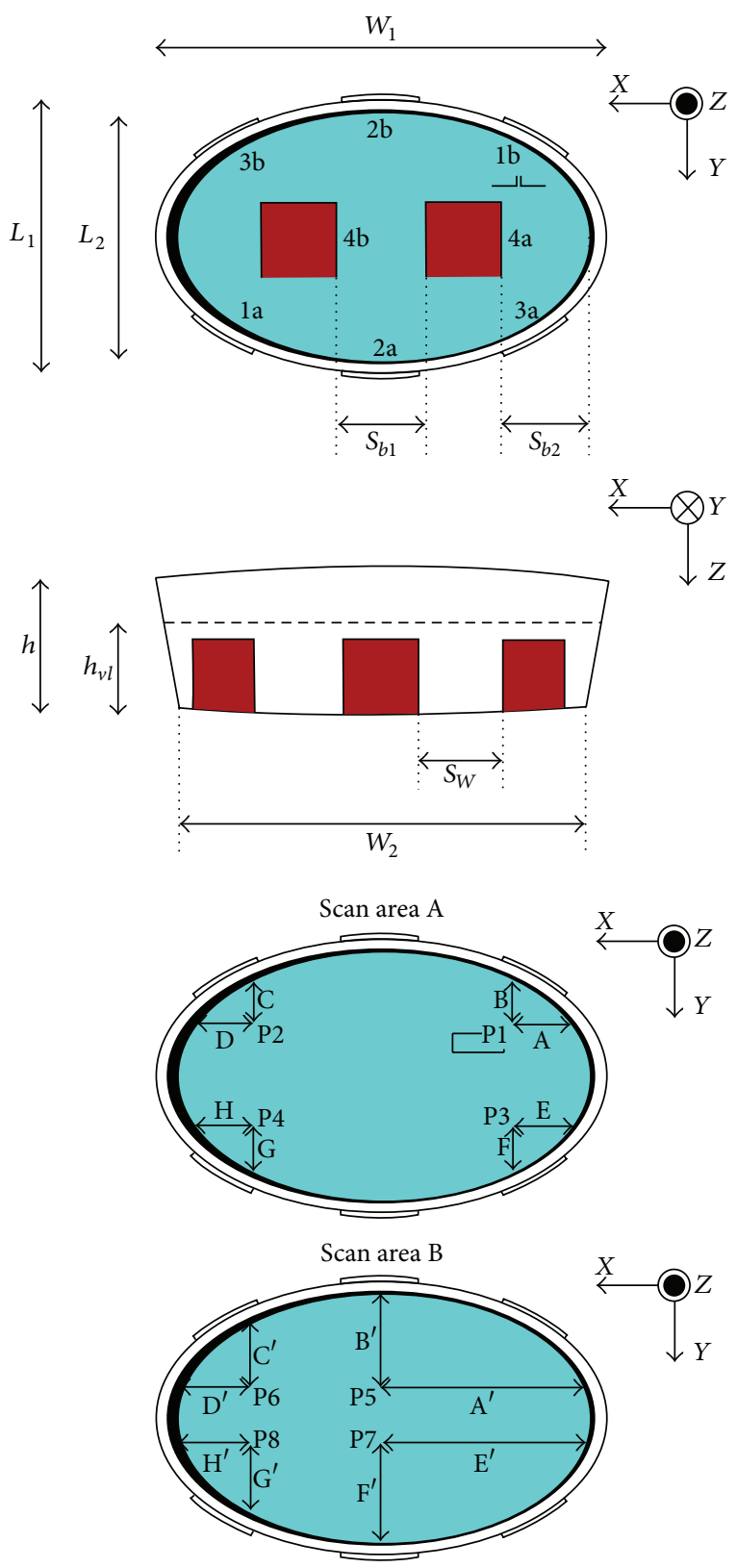

FIGURE 3: Human body phantom (top view, front view, scan area A for an $x$-and $y$-oriented dipole and scan area B for a $z$-oriented dipole).

$y$ orientation, area A was scanned, whereas for the vertical polarization, area B was scanned, both with a step size of $5 \mathrm{~mm}$. Each time the depth ( $z$-direction) corresponds to a dipole-center position $4.3 \mathrm{~cm}$ underneath the liquid surface and at $5.9 \mathrm{~cm}$ away from the bottom of the phantom. From the measurements along these 3 axes, we determine the minimum SNR received from an implant with constantly varying orientation, as occurring in WCE applications. The transmit power is $10 \mathrm{~mW}$, corresponding to half the allowed specific absorption rate (SAR) limit of $2 \mathrm{~W} / \mathrm{kg}$ averaged over $10 \mathrm{~g}$ tissue [IEC 62209].

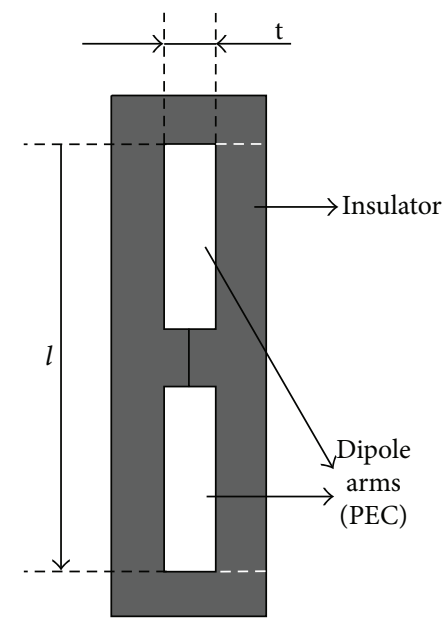

Figure 4: Transmitting insulated dipole $(t=1 \mathrm{~mm}, l=3.9 \mathrm{~cm})$.

TABLE 2: Statistical parameters of the stand-alone antennas (for an $x$-oriented dipole).

\begin{tabular}{ccccc}
\hline & $\begin{array}{c}\text { Max } \\
(\mathrm{dB})\end{array}$ & $\begin{array}{c}\text { Min } \\
(\mathrm{dB})\end{array}$ & $\begin{array}{c}\text { Mean } \\
(\mathrm{dB})\end{array}$ & $\begin{array}{c}\text { Median } \\
(\mathrm{dB})\end{array}$ \\
\hline $\mathrm{la}$ & 46.68 & -1.85 & 13.32 & 12.93 \\
$\mathrm{2a}$ & 24.96 & -1.22 & 12.84 & 13.32 \\
$\mathrm{3a}$ & 49.83 & -0.30 & 11.83 & 12.43 \\
$\mathrm{4a}$ & 41.87 & -1.83 & 12.46 & 9.04 \\
$\mathrm{lb}$ & 40.87 & -0.67 & 12.37 & 13.44 \\
$\mathrm{2b}$ & 24.57 & -0.32 & 12.88 & 12.33 \\
$\mathrm{3b}$ & 38.80 & -2.81 & 10.81 & 11.05 \\
$4 \mathrm{~b}$ & 38.91 & -1.25 & 11.12 & 6.98 \\
\hline
\end{tabular}

\section{Results and Discussion}

3.1. $x$-Oriented Dipole. The statistical parameters of the antennas, as viewed in Table 2, exhibit deep dips in the SNR for every single on-body receive antenna. These signal dips are much lower than the proposed $10 \mathrm{~dB}$ level, so, for a single wearable receive antenna, no optimal position can be determined on the surface of the human body phantom. By using multiple receive antennas, the probability that all antennas simultaneously receive weak signals strongly decreases. The maxima of the side-wall antennas $2 \mathrm{a}$ and $2 \mathrm{~b}$ are clearly below the maxima of the other six antennas. This is caused by the rectangular scan area in the oval phantom, where, due to the curvature of the ELI phantom, the middle antennas $2 \mathrm{a}$ and $2 \mathrm{~b}$ are located further away from the edges of the scan area, as shown in Figure 3.

The envelope correlation matrix for the signal levels, as presented in Table 3, demonstrates that all correlation coefficients are far below 0.7 , which indicates that the signals, received on the multiple antennas, are strongly uncorrelated, leading to a significant gain when employing spatial diversity [42]. The negative correlation coefficient between the bottom antennas $4 \mathrm{a}$ and $4 \mathrm{~b}$, equal to -0.04 , indicates that these two antennas are complementary. However, they only cover small 
TABLE 3: Envelope correlation matrix for the received signals (with $x$-oriented transmitting dipole).

\begin{tabular}{ccccccccc}
\hline & $1 \mathrm{a}$ & $3 \mathrm{~b}$ & $2 \mathrm{a}$ & $2 \mathrm{~b}$ & $3 \mathrm{a}$ & $1 \mathrm{~b}$ & $4 \mathrm{a}$ & $4 \mathrm{~b}$ \\
\hline $\mathrm{la}$ & 1.00 & 0.16 & 0.00 & -0.03 & -0.02 & -0.04 & -0.02 & 0.01 \\
$\mathrm{3b}$ & 0.16 & 1.00 & 0.01 & -0.01 & -0.01 & -0.04 & -0.03 & 0.03 \\
$\mathrm{2a}$ & 0.00 & 0.01 & 1.00 & 0.47 & 0.01 & -0.03 & -0.01 & -0.03 \\
$\mathrm{2b}$ & -0.03 & -0.01 & 0.47 & 1.00 & -0.01 & -0.03 & -0.04 & 0.02 \\
$3 \mathrm{a}$ & -0.02 & -0.01 & 0.01 & -0.01 & 1.00 & 0.14 & 0.01 & -0.03 \\
$\mathrm{lb}$ & -0.04 & -0.04 & -0.03 & -0.03 & 0.14 & 1.00 & 0.07 \\
$4 \mathrm{a}$ & -0.02 & -0.03 & -0.01 & -0.04 & 0.01 & 0.07 & -0.05 \\
$4 \mathrm{~b}$ & 0.01 & 0.03 & -0.03 & 0.02 & -0.03 & -0.05 & -0.04 & -0.04 \\
\hline
\end{tabular}

TABLE 4: Statistical parameters for all dipole orientations and varying diversity orders, where the gain is based on the $10 \%$ outage probability level of the CDF. Note the different scan area size for the $z$-orientation, due to physical constraints (Figure 3).

\begin{tabular}{|c|c|c|c|c|c|c|}
\hline $\begin{array}{l}\text { Dipole } \\
\text { orientation }\end{array}$ & $\begin{array}{c}\text { Diversity } \\
\text { order }\end{array}$ & $\begin{array}{l}\text { Max. } \\
(\mathrm{dB})\end{array}$ & $\begin{array}{l}\text { Min. } \\
(\mathrm{dB})\end{array}$ & $\begin{array}{c}\text { Mean } \\
(\mathrm{dB})\end{array}$ & $\begin{array}{l}\text { Median } \\
(\mathrm{dB})\end{array}$ & $\begin{array}{l}\text { Gain } \\
(\mathrm{dB})\end{array}$ \\
\hline \multirow{4}{*}{$X$} & 8 & 49.84 & 17.80 & 26.44 & 25.24 & 13.8 \\
\hline & 6 & 49.84 & 16.30 & 23.00 & 22.78 & 12.5 \\
\hline & 4 & 49.83 & 11.85 & 20.90 & 20.04 & 9.5 \\
\hline & 2 & 41.87 & 4.30 & 18.91 & 20.48 & 2.1 \\
\hline \multirow{4}{*}{$Y$} & 8 & 47.16 & 18.34 & 26.77 & 25.30 & 14.2 \\
\hline & 6 & 44.27 & 17.96 & 24.46 & 24.37 & 13.2 \\
\hline & 4 & 44.26 & 13.69 & 22.24 & 21.71 & 9.7 \\
\hline & 2 & 47.12 & 3.88 & 17.70 & 18.09 & 0.5 \\
\hline \multirow{4}{*}{$Z$} & 8 & 27.99 & 10.85 & 16.89 & 17.32 & 9.25 \\
\hline & 6 & 11.83 & 8.74 & 10.25 & 10.16 & 7.65 \\
\hline & 4 & 10.97 & 6.87 & 8.69 & 8.56 & 6.15 \\
\hline & 2 & 27.92 & 3.34 & 14.40 & 16.34 & 2.45 \\
\hline
\end{tabular}

nonoverlapping parts of the complete scan area. Hence, if 2nd order spatial diversity is applied based only on these two bottom antennas, the critical $10 \mathrm{~dB}$ SNR level will not be guaranteed for the complete area, as further described in Table 4 . In contrast to the bottom antennas $4 \mathrm{a}$ and $4 \mathrm{~b}$, the side-wall antennas $2 \mathrm{a}$ and $2 \mathrm{~b}$ have a significantly larger correlation coefficient, equal to 0.47 . As these two antennas are deployed at opposite sides, this means that these two side-wall antennas cover a significantly larger area of the oval phantom, with overlap of the covered regions. The correlation between, on the one hand, the side-wall antennas $2 \mathrm{a}$ and $2 \mathrm{~b}$ and, on the other hand, the four other side-wall antennas la, $3 \mathrm{a}, 1 \mathrm{~b}$, and $3 \mathrm{~b}$, is almost negligible. This indicates that by going from 4 th order spatial diversity, only considering corner side antennas $1 \mathrm{a}, 3 \mathrm{a}, \mathrm{b}$, and $3 \mathrm{~b}$, to 6 th order diversity, where the center side antennas $2 \mathrm{a}$ and $2 \mathrm{~b}$ are added to the diversity scheme, a significant gain will be obtained, as proven further. Since most correlation coefficients are low, mutual coupling between neighboring antennas is small.

By now evaluating an 8 th order receive diversity scheme and verifying if the design requirement of a minimal SNR larger than or equal to $10 \mathrm{~dB}$ is fulfilled, some conclusions can already be drawn. Figure 5 shows the SNR $(\mathrm{dB})$ as a function of position, for an $x$-oriented transmit dipole, applying 8 th-order diversity reception using maximal ratio combining (MRC).

Because of the fixed depth of the dipole, the perpendicular distance $d_{\text {bottom-dipole }}$ is equal to $5.9 \mathrm{~cm}$, leading to a large SNR received by the bottom antennas ( $4 \mathrm{a}$ and $4 \mathrm{~b}$ ) when the dipole is directly overhead. Note, however, that these antennas only cover a small region of the total scan area. Moreover, the SNR never drops below the critical $10 \mathrm{~dB}$ level, enabling live wireless video streaming. Hence, the setup with diversity order 8 , and an $x$-oriented dipole leads to a system that always ensures a sufficiently large SNR. Table 4 shows the relevant statistical figures of merit for this setup.

By reducing the diversity order from 8 to 6 , two main advantages arise. First, the patient comfort increases by removing the antennas on the back (antennas $4 \mathrm{a}$ and $4 \mathrm{~b}$ ) and, second, the cost of the wearable antenna system decreases. Figure 6 shows the SNR (dB) as a function of the position for 6 th order diversity with an $x$-oriented dipole.

As expected, by removing the bottom antennas that contribute only in a small area, the 6th order diversity system still satisfies the important $10 \mathrm{~dB}$ SNR design requirement. Table 4 shows the relevant statistical figures of merit for 8 th, 


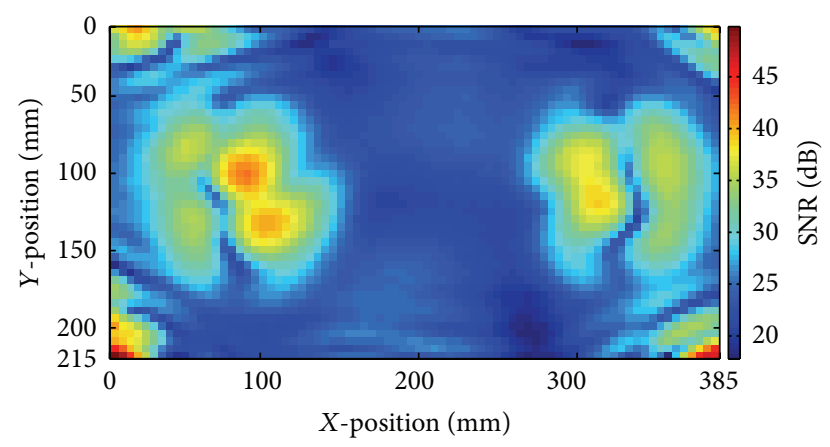

Figure 5: SNR (dB) as a function of the position for an $x$-oriented dipole (8th order diversity, all antennas included, scan area A).

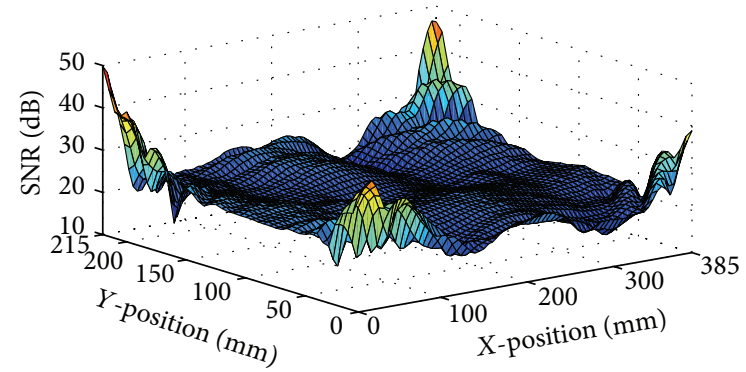

FIGURE 6: SNR (dB) as a function of the position for an $x$-oriented dipole (6th order diversity, bottom antennas $4 \mathrm{a}$ and $4 \mathrm{~b}$ excluded, scan area $\mathrm{A})$.

6 th, 4th, and 2nd order diversity systems. For 8 th order diversity, all antennas were considered, whereas for 6th order diversity antennas $4 \mathrm{a}$ and $4 \mathrm{~b}$ were excluded. For 4 th order diversity, only antennas $1 \mathrm{a}, 3 \mathrm{a}, 1 \mathrm{~b}$, and $3 \mathrm{~b}$ were considered and for 2 nd order diversity, only bottom antennas $4 \mathrm{a}$ and $4 \mathrm{~b}$ were taken into account.

By analyzing Table 4 for the $x$-oriented dipole, it is clear that a 2 nd order diversity system does not result in a reliable multiantenna system, as the minimal SNR equals $4.30 \mathrm{~dB}$, which is far below the $10 \mathrm{~dB}$ limit. By focusing further on the minimal SNR, some tendencies can be extracted from Table 4 . When increasing the diversity order from 4 to 6 , the minimal SNR increases by $4.45 \mathrm{~dB}$. This significant gain is obtained thanks to the inclusion of antennas $2 \mathrm{a}$ and $2 \mathrm{~b}$, which cover a large area. When further increasing the diversity order from 6 to 8 , the minimal SNR increases only by an extra $1.50 \mathrm{~dB}$. Including the bottom antennas $4 \mathrm{a}$ and $4 \mathrm{~b}$ in the 8 th-order diversity scheme only results in marginal improvements in diversity gain, as they only partly cover the scan area. The cumulative distribution function (CDF) of the obtained SNR gives an indication about the gain obtained by applying different orders of diversity. This CDF, corresponding to $P[X \leq \mathrm{SNR}(\mathrm{dB})]$, is shown in Figure 7 .

Figure 7 shows the CDFs for different orders of diversity. In order not to overload the plot, for the cases without diversity, realized by the single antennas, we only display the results for the bottom-antenna with the highest SNR (4a), for the best middle side-wall antenna (2a) and for the best

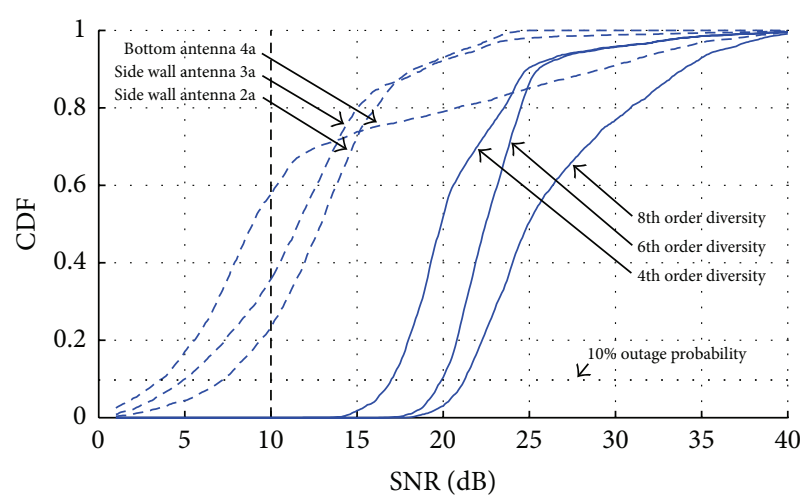

Figure 7: Cumulative distribution function (for an $x$-oriented dipole).

corner side-wall antenna (3a). The CDFs for the other five remaining antennas are shaped similarly to their counterparts located at equivalent positions. By focusing on the bestcase scenario, the minimal gain provided by implementing diversity is calculated.

Setting the criterion of an absolute minimum SNR of $10 \mathrm{~dB}$ guarantees continuous live video transmissions at an acceptable data rate without missing packets and without the need for a feedback link. However, the $10 \%$ outage probability level indicates the minimal SNR level and corresponding data rate that may be frequently obtained. On the condition that a channel feedback link is present, an endoscopy capsule can always use the currently available maximum data rate, which is controlled through feedback from the receiver. The in-body implant then needs to have memory to temporally buffer the recorded data, and provisions should be made to request retransmissions of potentially missed data packets. At the expense of higher power consumption and more complex hardware at both sides of the links, such a scheme enables transmissions of higher quality video signals, compared to a system without a feedback link.

The gains, obtained for different orders of diversity, are clearly visible as a shift to the right in the CDFs of different orders. Focusing on the $10 \%$ outage probability level, the 6 th order diversity performs $3 \mathrm{~dB}$ better than 4 th order diversity, and 8th order diversity performs $1.3 \mathrm{~dB}$ better than the 6 th order diversity system. The minimal gain in terms of $10 \%$ outage probability, when comparing antenna $2 \mathrm{a}$ (which has the best single-antenna signal behavior) to 4 th, 6 th, and 8 th order diversity, is $9.5 \mathrm{~dB}, 12.5 \mathrm{~dB}$, and 13.8 , respectively.

As seen in Figure 7, antennas $3 \mathrm{a}$ and $2 \mathrm{a}$ have an equally shaped CDF. However, the median of antenna $3 \mathrm{a}$ is $2 \mathrm{~dB}$ less than the median of antenna $2 \mathrm{a}$, which illustrates the larger coverage of the middle side-wall antennas $2 \mathrm{a}$ and $2 \mathrm{~b}$. The CDF of the bottom antenna 4a exhibits a high SNR for only a small part of the scanned area. The influence of these bottom antennas is clearly visible in the CDF of the 8th order diversity system, especially for the higher SNR values.

It is clear that, for an $x$-oriented dipole, the 4 th order multiantenna system still satisfies the proposed design requirement of the minimal SNR $\geq 10 \mathrm{~dB}$. Next, the results for 


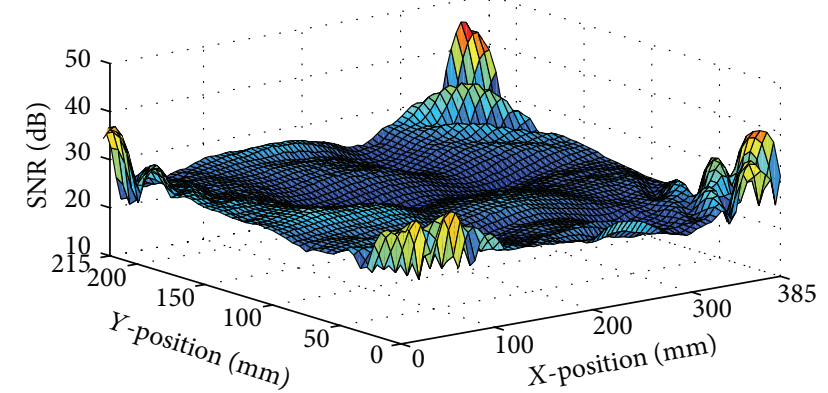

FIGURE 8: SNR (dB) as a function of the position for a $y$-oriented dipole (6th order diversity, bottom antennas $4 \mathrm{a}$ and $4 \mathrm{~b}$ excluded, scan area $\mathrm{A})$.

a $y$ - and $z$-oriented dipole are presented and compared with the previous results. From this, the minimal diversity order, guaranteeing high-reliability data communication for all potential dipole orientations, is derived.

3.2. $y$-Oriented Dipole. In the remainder, we focus on 8th, 6 th, and 4th order diversity. To make a comparison with the $x$-oriented dipole, Figure 8 shows the SNR (dB) as a function of the position, for a $y$-oriented transmitting dipole, with 6th order diversity reception applied.

Again, a 6th order multiantenna diversity system satisfies the requirement of a minimal SNR $\geq 10 \mathrm{~dB}$. Table 4 shows the statistical figures of merit, for an 8th, 6th, and 4th order diversity system in case of a $y$-oriented dipole. Again, a 4 th order diversity system provides a sufficiently large minimum SNR, more than $3 \mathrm{~dB}$ higher than the critical $10 \mathrm{~dB}$ limit. The CDFs, as well as the correlation matrix, show the same tendencies as in the case of an $x$-oriented dipole. When focusing on the $10 \%$ outage probability for the CDF, the minimal gain, when comparing reception by antenna $2 \mathrm{a}$ (which has again the best single-antenna behavior) to 4 th, 6th, and 8th order diversity, is equal to $9.7 \mathrm{~dB}, 13.2 \mathrm{~dB}$, and $14.2 \mathrm{~dB}$, respectively. Hence, the minimal gain for each diversity order is slightly higher than for an $x$-oriented dipole.

As in the previous situation for an $x$-oriented dipole, a diversity order of 6 seems to be optimal considering a balance between simplicity and/or cost, on the one hand, and highly reliable data links, on the other hand.

3.3. $z$-Oriented Dipole. Due to the small dimensions of the phantom, and the lengthy feeding part of the dipole, only a limited area B could be scanned for a vertically oriented dipole. This scan area B is shown in Figure 3. The most important region is in the middle because of the critical low SNR's that could occur when excluding the bottom antennas. Given the $x$ and $y$-symmetry of the phantom, conclusions can be extended to the complete phantom. Table 4 presents the statistical figures of merit for 8th, 6th and 4th order diversity in the case of a $z$-oriented dipole scanning the left-middle area of the bath (scan area B). The gain is again calculated for the $10 \%$ outage probability level but now compared to

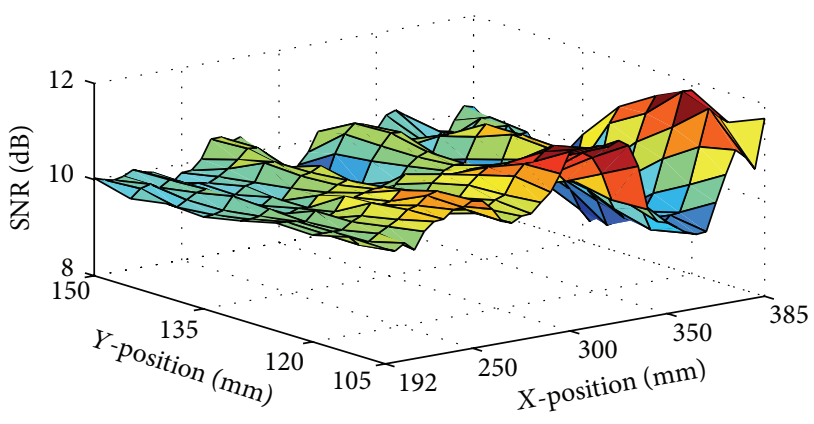

FIGURE 9: SNR (dB) as a function of position for a $z$-oriented dipole (6th order diversity, bottom antennas excluded, scan area B).

reception by antenna $3 b$, which has the best single antenna behavior for the $z$-oriented dipole.

Table 4 shows that 6 th order diversity does not ensure a sufficiently large SNR, due to the minimal SNR equal to $8.74 \mathrm{~dB}$, which indicates that, for a $z$-oriented dipole, 8th order diversity is necessary to guarantee a minimal SNR $\geq$ $10 \mathrm{~dB}$.

Because of the large influence of bottom antenna $4 \mathrm{~b}$ in scan area B, the bottom antennas are excluded in Figure 9, allowing more insight into the performance of the side-wall antennas. Figure 9 shows the SNR (dB) as a function of the position, for a $z$-oriented transmitting dipole, with 6th order diversity reception applied.

As Table 4 already indicated, the statistical values are below those of an $x$ - and $y$-oriented dipole for the same diversity order. This is because of the limited scan area $B$, where the distance to the side-wall antennas is large compared to scan area A.

For 6th order diversity, as presented in Figure 9, 34.2\% of the scan points are below the critical $10 \mathrm{~dB}$ SNR limit. However, the applied TX power at the implant is only half the allowed SAR limit [IEC 62209]; hence, when doubling the TX power to $20 \mathrm{~mW}$, the SNRs obtained with 6th order diversity increase by $3 \mathrm{~dB}$. In that case, the SNRs for the complete scan area would be above $10 \mathrm{~dB}$, with a minimum of $11.74 \mathrm{~dB}$.

\section{Conclusions}

A multiantenna system was developed for capturing high data-rate signals transmitted by implants. All antennas in this wearable system are flexible and fully fabricated using textile materials. Hence, the complete system can be easily and unobtrusively integrated into a jacket or another type of garment. The system is fully tested experimentally, by distributing the different antennas over the surface of a human body phantom, in order to determine the optimal antenna positions and the required diversity order for reliable high data rate communication with the implant. When limiting the TX power to $10 \mathrm{~mW}$, which is half the allowed SAR limit, 8th order spatial diversity is needed to allow live wireless video streaming with a bandwidth of $1 \mathrm{MHz}$ and a bit rate of $3.5 \mathrm{Mbit} / \mathrm{s}$. When doubling the TX power to $20 \mathrm{~mW}$, 6th order spatial diversity is sufficient to meet the imposed design 
requirements in all cases. This improves patient comfort and allows the use of the multiantenna system even when a patient is lying in a (hospital) bed, which could prove uncomfortable if two "back"-antennas are deployed in the patient's garment, as in case of 8th order diversity.

An important conclusion is that spatial diversity for an in-to-out body communication scenario allows the use of a simple transmit antenna in the implant, such as a single dipole-antenna. The on-body wearable multiantenna system then ensures a high-quality wireless link for any arbitrary orientation and position of the implant. In the perspective of wireless-endoscopy applications, the use of a simple antenna, operating at low power levels in the implanted capsule, decreases costs, size, and complexity of wireless camera capsule and increases its battery life.

\section{References}

[1] K. Kinsella and D. R. Phillips, "Global aging: the challenge of success," Population Bulletin, vol. 60, no. 1, pp. 3-40, 2005.

[2] M. E. Pollack, "Intelligent technology for an aging population: the use of AI to assist elders with cognitive impairment," $A I$ Magazine, vol. 26, no. 2, pp. 9-24, 2005.

[3] G. Iddan, G. Meron, A. Glukhovsky, and P. Swain, "Wireless capsule endoscopy," Nature, vol. 405, no. 6785, pp. 417-418, 2000.

[4] Z. Wang, E. G. Lim, T. Tillo, and F. Yu, "Review of the wireless capsule transmitting and receiving antennas," in Wireless Communications and Networks-Recent Advances, 2012.

[5] A. Karargyris and N. Bourbakis, "Three-dimensional reconstruction of the digestive wall in capsule endoscopy videos using elastic video interpolation," IEEE Transactions on Medical Imaging, vol. 30, no. 4, pp. 957-971, 2011.

[6] J. Hou, Y. Zhu, L. Zhang et al., "Design and implementation of a high resolution localization system for in-vivo capsule endoscopy," in Proceedings of the 8th IEEE International Symposium on Dependable, Autonomic and Secure Computing (DASC '09), pp. 209-214, Chengdu, China, December 2009.

[7] Y. Fan and M. Q.-H. Meng, "3D reconstruction of the WCE images by affine SIFT method," in Proceedings of the World Congress on Intelligent Control and Automation (WCICA '11), pp. 943-947, Tapei, Taiwan, June 2011.

[8] J. Thoné, S. Radiom, D. Turgis, R. Carta, G. Gielen, and R. Puers, "Design of a 2 Mbps FSK near-field transmitter for wireless capsule endoscopy," Sensors and Actuators A, vol. 156, no. 1, pp. 43-48, 2009.

[9] G. S. Lien, C. W. Liu, J. A. Jiang, C. L. Chuang, and M. T. Teng, "Magnetic control system targeted for capsule endoscopic operations in the stomach-design, fabrication, and in vitro and ex vivo evaluations," IEEE Transactions on Biomedical Engineering, vol. 59, no. 7, pp. 2068-2078, 2012.

[10] F. Carpi, N. Kastelein, M. Talcott, and C. Pappone, "Magnetically controllable gastrointestinal steering of video capsules," IEEE Transactions on Biomedical Engineering, vol. 58, no. 2, pp. 231-234, 2011.

[11] E. Y. Chow, M. M. Morris, and P. P. Irazoqui, "Implantable RF medical devices: the benefits of high-speed communication and much greater communication distances in biomedical applications," IEEE of Microwave Magazine, vol. 14, no. 4, pp. 64-73, 2013.
[12] Federal Communications Commission, "95. 627 MedRadio transmitters in the $401-406 \mathrm{MHz}$ band," in Code of Federal Regulations, Title 47-Telecommunications, Chapter 1-FCC, Subchapter D-Safety and Special Radio Services, Part 95-Personal Radio Services, Subpart E-Technical Regulations, 2012.

[13] European Telecommunications Standards Institute, "Electromagnetic compatibility and Radio spectrum Matters (ERM), Wideband transmission systems, Data transmission equipment operating in the $2,4 \mathrm{GHz}$ ISM band and using wide band modulation techniques," ETSI EN 300328 V1. 8. 1, 2012-04.

[14] X. Xie, G. Li, X. Chen, X. Li, and Z. Wang, "A low-power digital IC design inside the wireless endoscopic capsule," IEEE Journal of Solid-State Circuits, vol. 41, no. 11, pp. 2390-2400, 2006.

[15] B. Chi, J. Yao, S. Han, X. Xie, G. Li, and Z. Wang, "Low-power transceiver analog front-end circuits for bidirectional high data rate wireless telemetry in medical endoscopy applications," IEEE Transactions on Biomedical Engineering, vol. 54, no. 7, pp. 1291-1299, 2007.

[16] A. Khaleghi, R. Chávez-Santiago, X. Liang, I. Balasingham, V. C. M. Leung, and T. A. Ramstad, "On ultra wideband channel modeling for in-body communications," in Proceedings of the IEEE 5th International Symposium on Wireless Pervasive Computing (ISWPC '10), pp. 140-145, Modena, Italy, May 2010.

[17] A. Khaleghi and I. Balasingham, "Improving in-body ultra wideband communication using near-field coupling of the implanted antenna," Microwave and Optical Technology Letters, vol. 51, no. 3, pp. 585-589, 2009.

[18] S. Støa, R. Chavez-Santiago, and I. Balasingham, "An ultra wideband communication channel model for capsule endoscopy," in Proceedings of the 3rd International Symposium on Applied Sciences in Biomedical and Communication Technologies (ISABEL '10), pp. 1-5, Rome, Italy, November 2010.

[19] Q. Wang, K. Masami, and J. Wang, "Channel modeling and BER performance for wearable and implant UWB body area links on chest," in Proceedings of the IEEE International Conference on Ultra-Wideband (ICUWB '09), pp. 316-320, Vancouver, Canada, September 2009.

[20] A. Ghildiyal, K. Amara, R. D. Molin, B. Godara, A. Amara, and R. K. Shevgaonkar, "UWB for in-body medical implants: a viable option," in Proceedings of the IEEE International Conference on Ultra-Wideband (ICUWB '10), pp. 450-453, Nanjing, China, September 2010.

[21] J. Shi and J. Wang, "Channel characterization and diversity feasibility for in-body to on-body communication using lowband UWB signals," in Proceedings of the 3rd International Symposium on Applied Sciences in Biomedical and Communication Technologies (ISABEL '10), pp. 1-4, Rome, Italy, November 2010.

[22] J. Shi, D. Anzai, and J. Wang, "Diversity performance of UWB LOW band communication over In-body to On-body Propagation Channel," in Proceedings of the 6th European Conference on Antennas and Propagation (EUCAP '12), pp. 535-539, Prague, Czech Republic, March 2012.

[23] Y. Luo, C. Winstead, and P. Chiang, "125 Mbps ultra-wideband system evaluation for cortical implant devices," in Proceedings of the 34th Annual International Conference of the IEEE EMBS, pp. 779-782, San Diego, Calif, USA, August 2012.

[24] A. Ghildiyal, B. Godara, K. Amara, R. D. Molin, and A. Amara, "Ultra Wideband for in and on-body medical implants: a study of the limits and new opportunities," in Proceedings of the 5th European Conference on Antennas and Propagation (EUCAP '11), pp. 3778-3782, Rome, Italy, April 2011. 
[25] J. Wang and Q. Wang, "Channel modelling and BER performance of an implant UWB body area link," in Proceedings of the 2nd International Symposium on Apllied Sciences in Biomedical and Communication Technologies, pp. 1-4, November 2009.

[26] European Telecommunications Standards Institute, "Electromagnetic compatibility and Radio spectrum Matters (ERM), Ultra Low Power Active Medical Implants (ULP-AMI) operating in the $401 \mathrm{MHz}$ to $402 \mathrm{MHz}$ and $405 \mathrm{MHz}$ to $406 \mathrm{MHz}$ bands," System Reference Document 102343 v1.1.1, 2004-07.

[27] International Electrotechnical Commission, "Human exposure to radio frequency fields from hand-held and body-mounted wireless communication devices-human body models," Instrumentation and Procedures, IEC62209 Standard, 2010.

[28] T. Karacolak, A. Z. Hood, and E. Topsakal, "Design of a dualband implantable antenna and development of skin mimicking gels for continuous glucose monitoring," IEEE Transactions on Microwave Theory and Techniques, vol. 56, no. 4, pp. 1001-1008, 2008.

[29] F. Merli, L. Bolomey, J. Zürcher, G. Corradini, E. Meurville, and A. K. Skrivervik, "Design, realization and measurements of a miniature antenna for implantable wireless communication systems," IEEE Transactions on Antennas and Propagation, vol. 59, no. 10, pp. 3544-3555, 2011.

[30] A. Alomainy and Y. Hao, "Modeling and characterization of biotelemetric radio channel from ingested implants considering organ contents," IEEE Transactions on Antennas and Propagation, vol. 57, no. 4, pp. 999-1005, 2009.

[31] Y. Chan, M. Q.-H. Meng, K.-L. Wu, and X. Wang, "Experimental study of radiation efficiency from an ingested source inside a human body model," in Proceedings of the 27th Annual International Conference of the Engineering in Medicine and Biology Society (IEEE-EMBS '05), pp. 7754-7757, Shangai, China, September 2005.

[32] A. Alomainy, Y. Hao, Y. Yuan, and Y. Liu, "Modelling and characterisation of radio propagation from wireless implants at different frequencies," in Proceedings of the 9th European Conference on Wireless Technology (ECWT '06), pp. 119-122, Manchester, UK, September 2006.

[33] A. Sani, A. Alomainy, and Y. Hao, "The effect of various human body tissue models on radiowave propagation from a bladder implanted wireless source," in Proceedings of the 2009 IEEE International Symposium on Antennas and Propagation and USNC/URSI National Radio Science Meeting (APSURSI '09), South Carolina, SC, USA, June 2009.

[34] W. G. Scanlon, J. Brian Burns, and N. E. Evans, "Radiowave propagation from a tissue-implanted source at $418 \mathrm{MHz}$ and 916.5 MHz," IEEE Transactions on Biomedical Engineering, vol. 47, no. 4, pp. 527-534, 2000.

[35] D. Kurup, W. Joseph, G. Vermeeren, and L. Martens, "Inbody path loss model for homogeneous human tissues," IEEE Transactions on Electromagnetic Compatibility, vol. 54, no. 3, pp. 556-5564, 2012.

[36] D. Kurup, W. Joseph, E. Tanghe, G. Vermeeren, and L. Martens, "Extraction of antenna gain from path loss model for in-body communication," Electronics Letters, vol. 47, no. 23, pp. 12621263, 2011.

[37] P. D. Bradley, "An ultra low power, high performance Medical Implant Communication System (MICS) transceiver for implantable devices," in Proceedings of the IEEE Biomedical Circuits and Systems Conference Healthcare Technology (BioCAS '06), pp. 158-161, London, UK, December 2006.
[38] D. Andreuccett, R. Fossi, and P. Caterina, "Calculation of the dielectric properties of body tissues in the frequeny range $10 \mathrm{~Hz}-100 \mathrm{GHz}$," Italian National Research Council- Institute for Applied Physics, Daniele Andreuccetti, Roberto Fossi and Caterina Petrucci http://niremf.ifac.cnr.it/tissprop/htmlclie/ htmlclie.htm\#atsftag, 1997.

[39] S. Agneessens, P. Van Torre, E. Tanghe, G. Vermeeren, W. Joseph, and H. Rogier, "On-body wearable repeater as a data link relay for in-body wireless implants," IEEE Antennas and Wireless Propagation Letters, vol. 11, pp. 1714-11717, 2012.

[40] G. Ciuti, A. Menciassi, and P. Dario, "Capsule endoscopy: from current achievements to open challenges," IEEE Reviews in Biomedical Engineering, vol. 4, pp. 59-72, 2011.

[41] L. Vallozzi, P. Van Torre, C. Hertleer, H. Rogier, M. Moeneclaey, and J. Verhaevert, "Wireless communication for firefighters using dual-polarized textile antennas integrated in their garment," IEEE Transactions on Antennas and Propagation, vol. 58, no. 4, pp. 1357-1368, 2010.

[42] S. M. Alamouti, "A simple transmit diversity technique for wireless communications," IEEE Journal on Selected Areas in Communications, vol. 16, no. 8, pp. 1451-1458, 1998. 

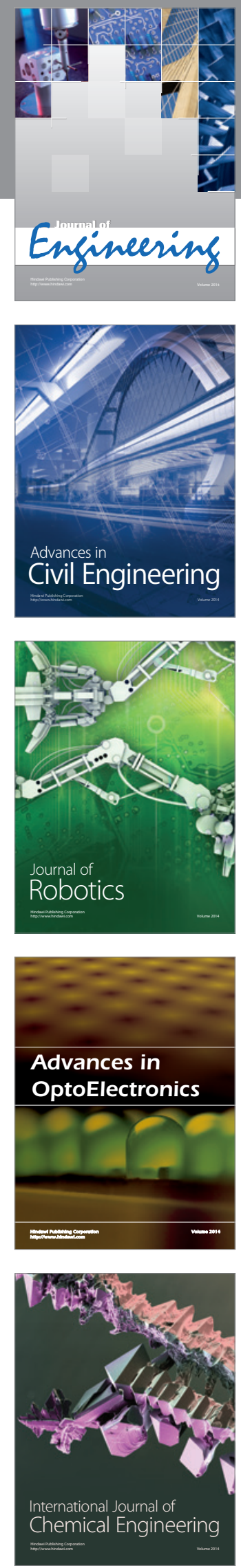

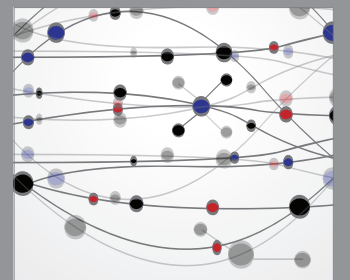

The Scientific World Journal
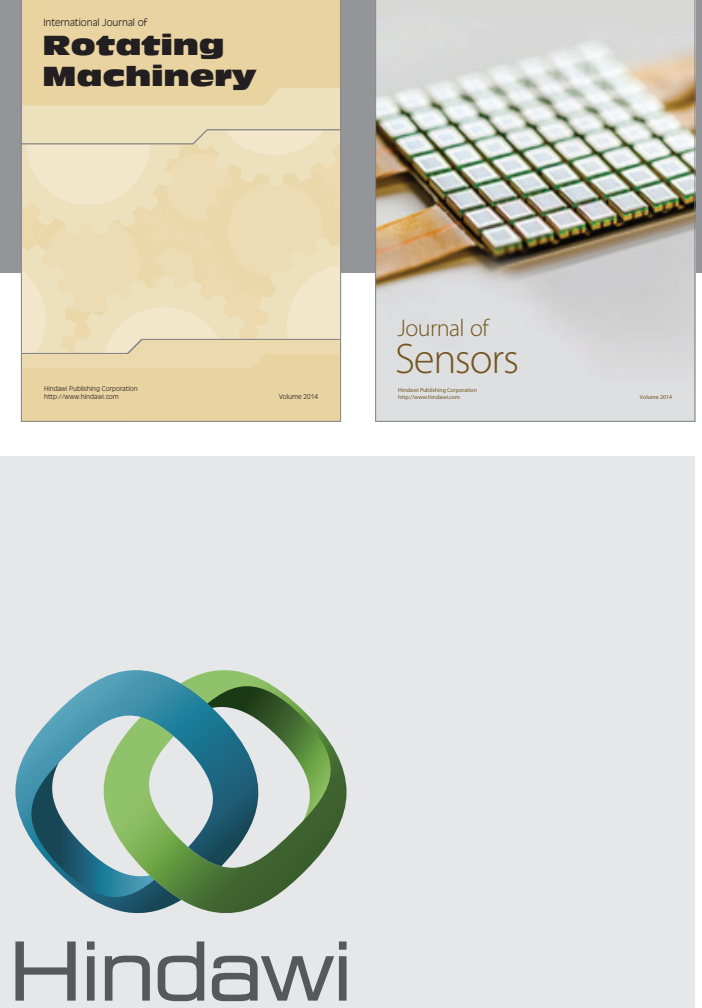

Submit your manuscripts at http://www.hindawi.com
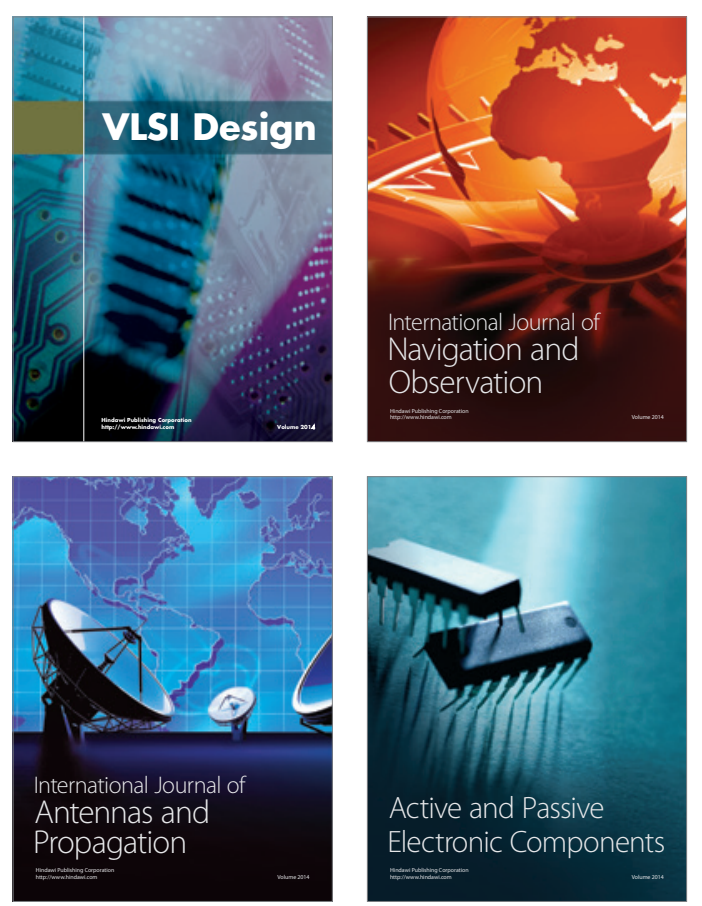
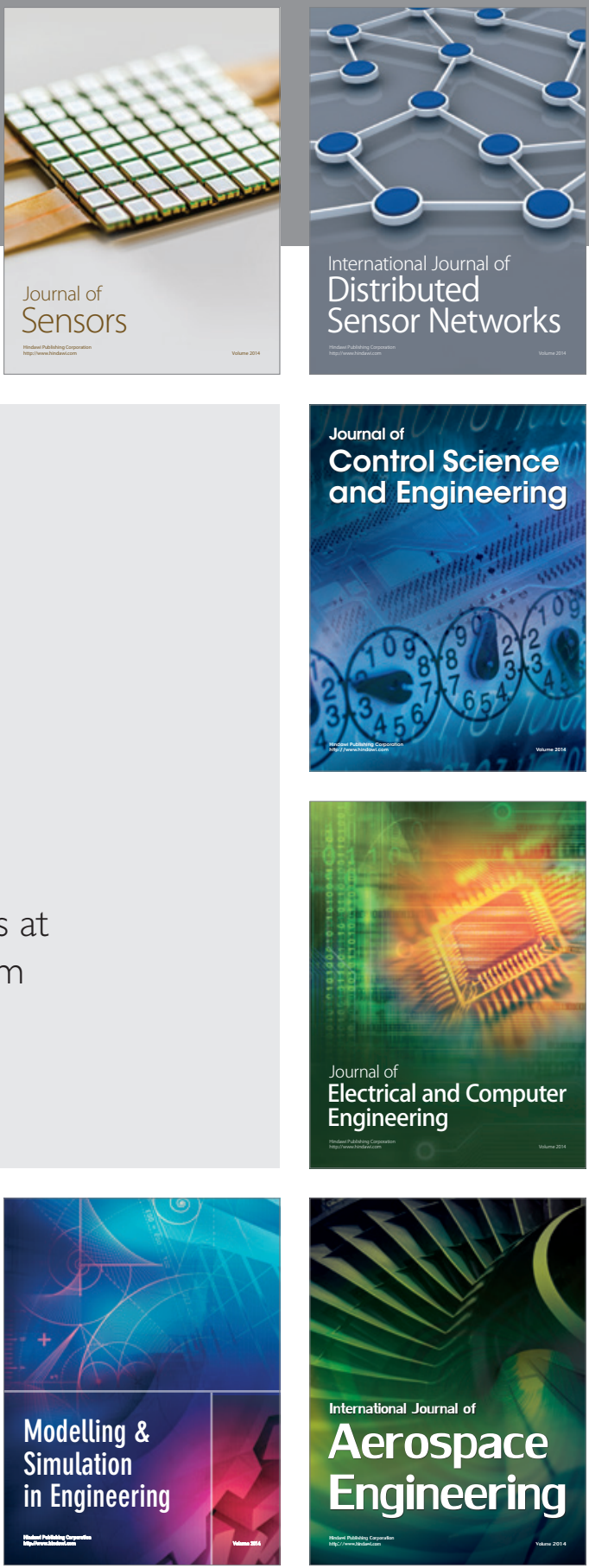

Journal of

Control Science

and Engineering
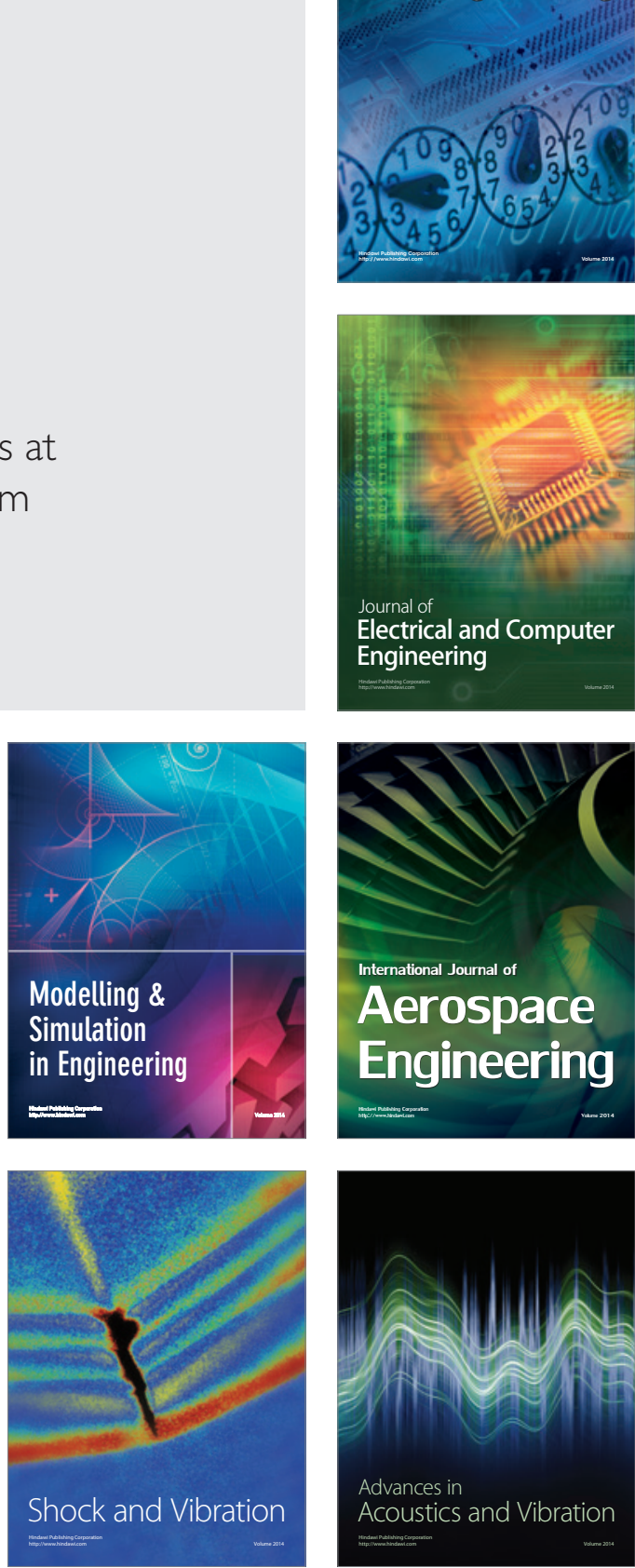